\title{
HIV-/Aidsepidemie: Präventionsarbeit durch Hausärztinnen und Hausärzte in der Schweiz
}

\author{
G. Meystre-Agustoni, A. Jeannin, F. Dubois-Arber
}

Im Auftrag des BAG dokumentiert das Institut für Sozial- und Präventivmedizin der Universität Lausanne bereits zum dritten Mal seit 1990 das Engagement der Schweizer Ärztinnen und Ärzte bezüglich HIV-Prävention bei Konsultationen in der Privatpraxis. Die neuste Erhebung, realisiert im Jahr 2002, stellt eine Verbesserung der Risikoanamnese insbesondere bei Patientinnen und Patienten aus der Allgemeinbevölkerung fest. Die Studie kommt jedoch zum Schluss, dass nach wie vor ein grosses Verbesserungspotential besteht - speziell bei jungen Erwachsenen, neuen Patienten sowie Migranten.

\section{Einleitung}

Die Beziehung zwischen Arzt und Patient eignet sich besonders gut, um individuell abgestimmte Ratschläge im Bereich der Prävention abzugeben. Auf Grund der grossen Häufigkeit der Konsultationen, die in der Schweiz verzeichnet werden, weist diese Begegnung ein noch höheres Potential auf. Daher betrachtet das Bundesamt für Gesundheit (BAG) die Prävention in der Arztpraxis [1-4] als zentralen Pfeiler seiner Strategie zur Bekämpfung von HIV/Aids. Es ist bestrebt, die Präventionsarbeit der Ärztinnen und Ärzte zu unterstützen und zu fördern. Diese Präventionstätigkeit wurde 1990 und 1995 evaluiert [5, 6]. Eine weitere Evaluation fand 2002 statt. Sie ermöglicht es, sich ein Bild von der Entwicklung der Situation zu machen. Die Studie zeigt namentlich, wie die in einer Privatpraxis tätigen Ärztinnen und Ärzte ihre Rolle im Zusammenhang mit der Epidemie wahrnehmen. Zudem ermöglicht sie es, die Präventionsaktivitäten zu beschreiben und mit jenen zu vergleichen, die 1990 und 1995 beobachtet wurden.

\section{Methoden}

a In diesem Fachgebiet wurden alle in der Schweiz tätigen Ärztinnen und Ärzte berücksichtigt.

Korrespondenz: Giovanna Meystre-Agustoni Institut für Sozial- und Präventivmedizin Rue du Bugnon 17 CH-1005 Lausanne logen ${ }^{\text {a }}$. Die definitive Stichprobe umfasste 2018 frei praktizierende Ärztinnen und Ärzte.

Für die 1990 und 1995 durchgeführten Studien wurden die nach Sprachregion und Fachgebiet geschichteten Stichproben nach einer analogen Methode zusammengestellt. Allerdings waren die Infektiologen darin nicht enthalten. In diesen Studien hatten 702 (63\%) beziehungsweise 796 (55\%) Ärztinnen und Ärzte geantwortet.

Wie in den früheren Evaluationen wurden die Daten mittels eines anonymen, per Post versandten Fragebogens erhoben. Um die Entwicklung im Verlauf der Zeit zu erfassen, waren einige Fragen bezüglich der Präventionstätigkeit identisch mit den Fragen, die bereits 1990 und 1995 gestellt worden waren. Es wurden namentlich die folgenden Bereiche untersucht:

- Einstellung der Ärztinnen und Ärzte gegenüber der HIV-/Aidsprävention und gegenüber bestimmten ethischen Problemen;

- Anamnese und Risikoanamnese (Patiententypen, systematische Durchführung);

- Beratung zum Zeitpunkt des HIV-Tests (Häufigkeit und Inhalt).

Die Rücklaufquote lag bei 61\% (1231 Ärztinnen und Ärzte sandten den Fragebogen zurück).

Die Trendanalysen beziehen sich nach Möglichkeit auf zwölf Jahre. Da die Infektiologen in den früheren Studien nicht enthalten waren, wurden sie nicht berücksichtigt.

\section{Resultate}

Damit hochstehende Präventionsleistungen angeboten werden können, die auf die Situation und die spezifischen Bedürfnisse der einzelnen Patientinnen und Patienten abgestimmt sind, muss deren Situation in bezug auf das HIV-Risiko abgeklärt werden. Besondere Beachtung ist der Anamnese und vor allem jenen Themen zu schenken, die sich auf eine allfällige HIV-Exposition beziehen.

Fast alle Ärztinnen und Ärzte (90\%) erachten es als «sehr wichtig» oder «wichtig», im HIV-/ Aidsbereich weiterhin präventiv tätig zu sein. 
Abbildung 1

Oft oder immer angesprochene Themen bei der Anamnese von jungen Erwachsenen $(n=1231)$. Hinweis: Vertrauensintervalle bei 95\% unter Annahme einer Normalverteilung.

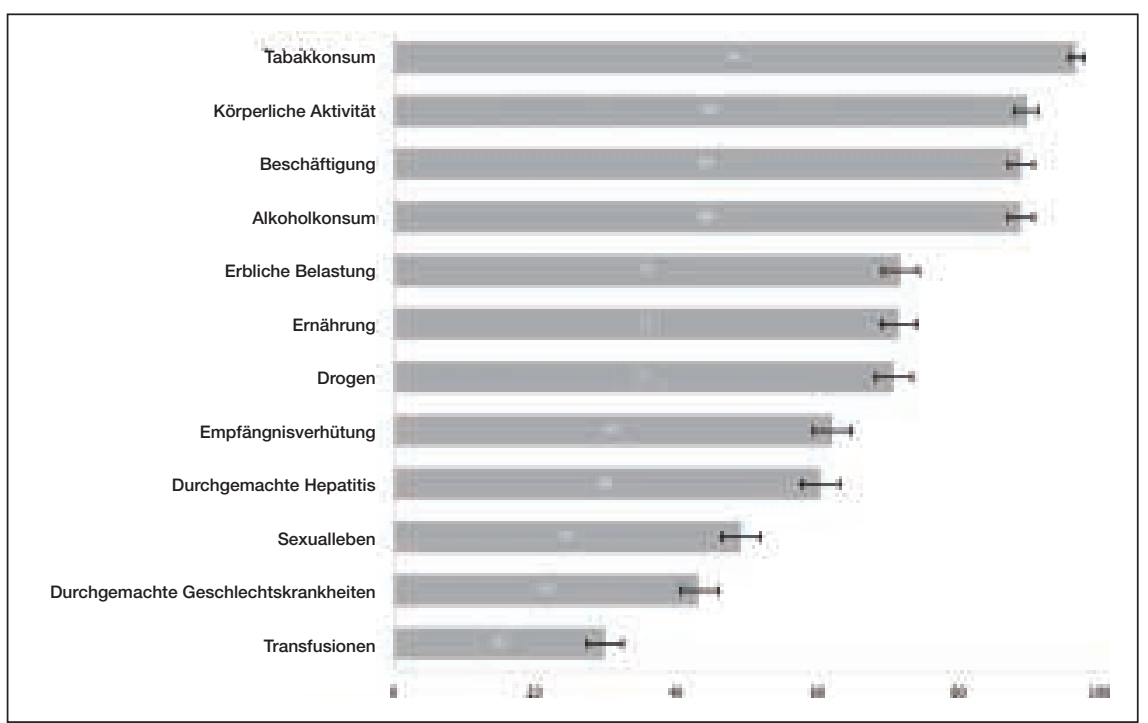

Allerdings sind 12\% mit folgender Aussage «einverstanden» oder «eher einverstanden»: «Der Wissensstand der Patientinnen und Patienten ist bereits ausreichend, so dass es keiner zusätzlichen Präventionsarbeit seitens der Ärzteschaft bedarf.» Diese Anteile entsprechen in etwa jenen, die 1990 und 1995 festgestellt wurden.

\section{Eine auf die Gesamtbevölkerung ausgerichtete Präventionspraxis}

Den Ärztinnen und Ärzten wurde die Frage gestellt, was sie im Fall von jungen Erwachsenen unternehmen würden, die ihre Praxis wegen eines nicht dringlichen Gesundheitsproblems aufsuchen. Abbildung 1 zeigt, dass sie sich systematisch nach dem Tabak- und Alkoholkonsum, der körperlichen Aktivität und der Berufstätigkeit erkundigen würden (rund 90\% der Ärztinnen und Ärzte). Hingegen werden bestimmte Themen, die auf eine HIV-Exposition hinweisen können, weniger häufig angesprochen. So erkundigen sich nur zwei Drittel der Ärztinnen und Ärzte immer oder oft nach einer allfälligen Drogenabhängigkeit ihres Patienten/ihrer Patientin oder nach der Verhütungsmethode (71 bzw. 62\%). Schliesslich würde nur die Hälfte (48\%) routinemässig eine Sexualanamnese durchführen. Im Vergleich zu den $1995^{\mathrm{b}}$ erhobenen Daten haben sich die Gewohnheiten der Ärztinnen und Ärzte praktisch nicht verändert.

Wenn die Ärztinnen und Ärzte eine Sexualanamnese vornehmen, richten sie ihre Aufmerksamkeit vor allem auf den Gebrauch von Präservativen $(84 \%)$ sowie auf die auftretenden Probleme (70\%). Rund die Hälfte der Ärztinnen und Ärzte erkundigt sich immer oder oft nach der sexuellen Ausrichtung des Patienten sowie nach der Zahl seiner Partner (57 bzw. 49\%). Schliesslich dehnt ein Drittel der Ärztinnen und Ärzte (33\%) die Anamnese immer oder oft auf die Sexualpraktiken aus. Auch diesbezüglich haben die Ärztinnen und Ärzte ihre Gewohnheiten seit 1995 praktisch nicht verändert.

Um die Vorgehensweisen im Bereich der Anamnese des HIV-Risikos zu evaluieren, wurden im Fragebogen verschiedene Typen von Patientinnen/Patienten und Situationen aufgeführt. Die Ärztinnen und Ärzte reagieren unterschiedlich auf die vorgeschlagenen Situationen und Patientenprofile. In einigen Fällen ist der Anteil jener, die sich nach der Möglichkeit einer HIV-Exposition erkundigen würden, bis zu dreimal höher als in anderen Fällen. Aufmerksamkeit erhalten in erster Linie jene Patientinnen/Patienten oder Situationen, die explizit auf die Möglichkeit eines Kontakts mit HIV verweisen (Drogenabhängigkeit, Sexualleben). Patientinnen/Patienten und Situationen, die nicht direkt auf HIV verweisen (junge Erwachsene, Check-up, neue Patienten) werden zwar nicht vernachlässigt, lösen jedoch weniger oft eine Risikoanamnese aus. Schliesslich werden Menschen mit Migrationshintergrund und Patientinnen/Patienten, die sich von ihrem Partner getrennt haben, weniger häufig als die anderen Patienten entsprechend befragt (Abb. 2).

Das Verhalten der Ärztinnen und Ärzte in bezug auf die anamnestische Erfassung einer allfälligen HIV-Exposition hat sich im Verlauf der Jahre verändert. 1990 richteten die Ärztinnen und Ärzte ihre Aufmerksamkeit vor allem auf jene Gruppen, die klassischerweise als gefährdet betrachtet wurden. Zwölf Jahre danach schenken sie der Situation der Gesamtbevölkerung (Schwangerschaft, Anfrage wegen Verhütungsmitteln, junge Erwachsene, neue Patientinnen/Patienten und - in geringerem Ausmass - Menschen mit Migrationshintergrund) deutlich mehr Beachtung. In bezug auf die Risikoanamnese bei homosexuellen Patienten ist hingegen zwischen 1995 und 2002 ein leichter Rückgang festzustellen (Abb. 2).

Der HIV-Test bietet Gelegenheit, die Kenntnisse der Patientin/des Patienten in bezug auf die Übertragungswege zu ermitteln. Ausserdem kann in diesem Zusammenhang versucht werden, die Risiken zu erfassen, denen sich der Patient aussetzt (oder ausgesetzt hat), und der Patient kann bezüglich des Schutzverhaltens beraten und auf die begrenzte Aussagekraft des Tests aufmerksam gemacht werden («diagnostisches Fenster»). Die Studie zeigt jedoch, dass 
nur dieses letztere Thema (diagnostisches Fenster) von über der Hälfte der Ärztinnen und Ärzte systematisch angesprochen wird. Dagegen gehen weiterhin über 10\% der Ärztinnen und Ärzte nicht oder nur selten auf dieses Thema ein. Ausserdem ermittelt ein Viertel der Ärztinnen und Ärzte die Kenntnisse der Patientin/des Patienten gar nicht oder nur selten, und ein Fünftel würde nicht oder nur selten versuchen, die Risiken zu eruieren, denen sich ihr Patient aus-

\section{Abbildung 2}

Systematische Anamnese («oft» oder «immer») des HIV-Risikos bei verschiedenen Typen von Patienten und Situationen: Vergleich zwischen $1990(n=702), 1995(n=796)$ und 2002 ( $n=1217$, ohne Infektiologen).

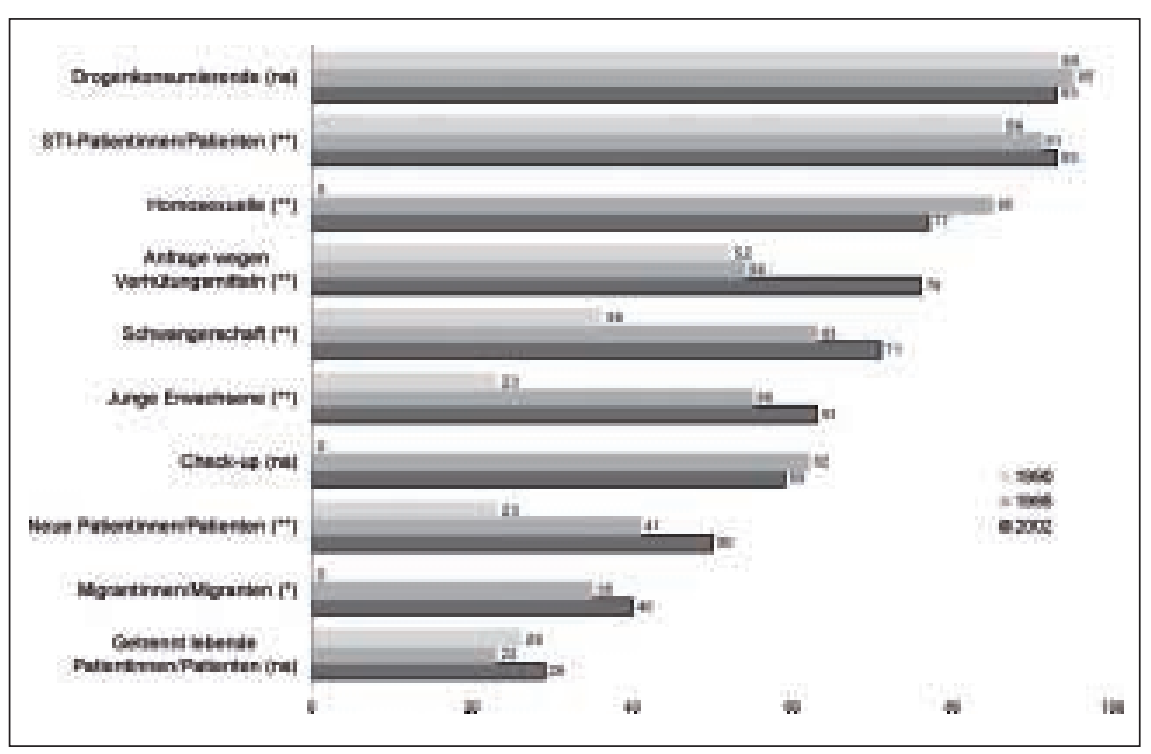

ns $=$ nicht signifikant $\quad{ }^{*}=p<.01 \quad * *=p<.001$

Abbildung 3

Beratung im Zusammenhang mit dem HIV-Test: Häufigkeit der angesprochenen Themen $(n=1231)$.

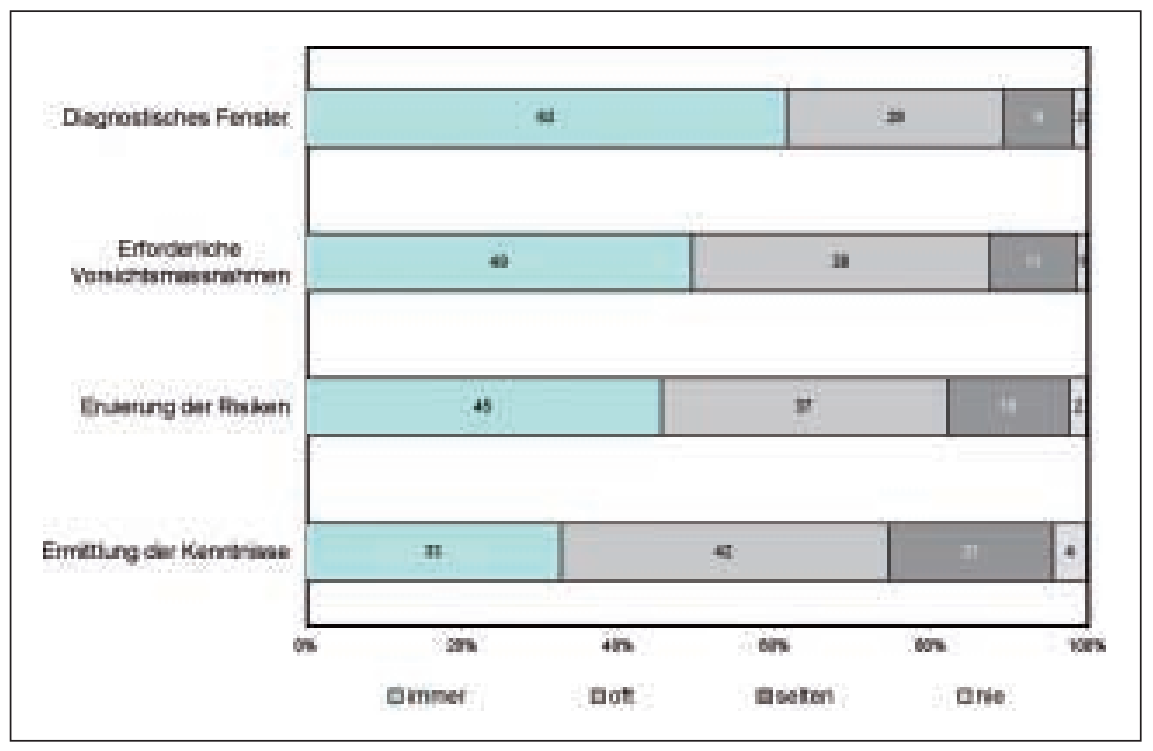

c Ein Vergleich mit 1990 ist nicht möglich. setzt. Schliesslich gibt nur die Hälfte der Ärztinnen und Ärzte systematisch Ratschläge ab, welche Vorsichtsmassnahmen zu treffen sind, um eine Infektion zu verhindern, während $12 \%$ dies selten oder nie tun (Abb. 3). Die Häufigkeit der verschiedenen Themen, die von den Ärztinnen und Ärzten anlässlich des Tests angesprochen werden, hat sich zwischen 1995 und 2002 nicht verändertc ${ }^{c}$.

\section{Diskussion}

Trotz der therapeutischen Fortschritte ist die HIV-/Aidsprävention noch immer von zentraler Bedeutung, und die Gesundheitsbehörden erwarten von den Ärztinnen und Ärzten weiterhin ein besonderes Engagement für eine individuell abgestimmte Beratung [3]. Diese Beratung erfordert eine detaillierte Erfassung der Risiken, denen sich die Patientin/der Patient möglicherweise ausgesetzt hat. Dazu sollen im Rahmen einer Anamnese die Themen im Zusammenhang mit der HIV-Übertragung (Sexualleben, intravenöser Drogenkonsum) angesprochen werden. Gegebenenfalls ist ein HIV-Test vorzuschlagen.

2002 betrachten sich die meisten frei praktizierenden Schweizer Ärztinnen und Ärzte als wichtige Akteure der HIV-/Aidsprävention. Diesbezüglich ist kein Rückgang gegenüber den früheren Evaluationen (1990 und 1995) festzustellen. In der Praxis stellen die Ärztinnen und Ärzte jedoch wie in den Vorjahren weiterhin Themen wie den Tabak- und Alkoholkonsum oder die körperliche Aktivität in den Vordergrund und achten noch zuwenig auf jene Themen, mit denen allfällige HIV-Expositionen aufgedeckt werden könnten. Bei jungen Erwachsenen führt nur die Hälfte der Ärztinnen und Ärzte eine Sexualanamnese durch. Die 2002 erhobenen Daten zeigen überdies, dass diese Art von Befragung häufig nur lückenhaft erfolgt. Während die verwendete Schutzmethode bei Sexualkontakten häufig erhoben wird, werden andere Themen seltener angesprochen, obwohl sie wichtig sind, um das Risiko einer HIV-Exposition zu beurteilen (sexuelle Ausrichtung, Anzahl Partner, Sexualpraktiken).

Im Vergleich zu 1990 ist bei den Schweizer Ärztinnen und Ärzten eine deutliche Verbesserung in bezug auf die Analyse des eigentlichen HIV-Risikos zu beobachten. In diesem Rahmen wird heute Personen und Situationen aus der Gesamtbevölkerung wie jungen Erwachsenen, neuen Patientinnen/Patienten, Schwangerschaften oder Anfragen wegen Verhütungsmitteln mehr Aufmerksamkeit geschenkt. Trotzdem 
wird weiterhin ein relativ grosses Präventionspotential nicht genutzt. Dies gilt insbesondere bei jungen Erwachsenen, neuen Patienten und Menschen mit Migrationshintergrund. Schliesslich bestehen noch immer Lücken im Bereich der Beratung vor dem Test. Wie auch aus anderen Studien hervorgeht [7-9], werden in diesem Rahmen noch längst nicht systematisch die Kenntnisse der Patientin/des Patienten ermittelt, die Risiken eruiert und eine Beratung bezüglich der erforderlichen Vorsichtsmassnahmen durchgeführt.

Bei einem Vergleich mit den Daten anderer Länder ist grosse Vorsicht geboten. In einem Umfeld, in dem sich die Erkenntnisse und therapeutischen Ressourcen rasch weiterentwickeln, kann schon die Differenz weniger Jahre zwischen den Datenerhebungen von grosser Bedeutung sein. Zudem sollten die Bedingungen berücksichtigt werden, unter denen die Ärztinnen und Ärzte ihre Tätigkeit ausüben. Denn diese Bedingungen können je nach Land sehr unterschiedlich sein und die Leistungen beeinflussen, die für die Patientinnen/Patienten erbracht werden. Im Ausland ist das Engagement der Ärztinnen und Ärzte hinsichtlich einer Sexualanamnese bei ihren Patientinnen/Patienten sehr unterschiedlich. In den Vereinigten Staaten stellten Ashton et al. [10] fest, dass sich 88\% der frei praktizierenden Ärztinnen und Ärzte im Bundesstaat Pennsylvania für die sexuelle Aktivität ihre Patientinnen/Patienten im Alter von 15 bis 25 Jahren interessieren, und gemäss Torkko et al. [11] geben $67,7 \%$ der freipraktizierenden Ärztinnen und Ärzte im Bundesstaat Colorado an, eine Sexualanamnese durchzuführen, wenn sie von einer Jugendlichen konsultiert werden $(90,1 \%$ der Gynäkologen, jedoch nur $43,9 \%$ der Internisten). Ellen et al. [12] stellten bei einer Gruppe von Ärztinnen und Ärzten aus den Vereinigten Staaten fest, dass 40\% eine Sexualanamnese durchführen. Bei kanadischen Ärztinnen und Ärzten zeigten Haley et al. [13] sowie Maheux et al. [14] auf, dass (je nach Fachgebiet) höchstens 40\% versuchen, die im Sexualbereich eingegangenen Risiken zu beurteilen. Schliesslich stellten Ewing et al. [15] fest, dass 24\% der amerikanischen Ärztinnen und Ärzte die sexuelle Aktivität ihrer Patientinnen/ Patienten routinemässig evaluieren.

Wie dies auch bei den frei praktizierenden Ärztinnen und Ärzten in der Schweiz festgestellt wurde, ist der Inhalt der Sexualanamnese unterschiedlich: Sie wird mehr oder weniger umfassend durchgeführt, und bestimmte Themen werden weniger häufig angesprochen als andere [12, 16-19].
Zusammenfassend lässt sich die Situation in der Schweiz als insgesamt gut, aber noch verbesserungsfähig bezeichnen. Nicht vernachlässigt werden darf jedoch die Gefahr, dass die Bevölkerung die Epidemie teilweise unterschätzt [20-22]. Die Ärztinnen und Ärzte müssen ihre Präventionsarbeit konsolidieren oder gar ausbauen, um zum einen Neuinfektionen zu verhindern und zum anderen den Anteil der seropositiven Personen zu erhöhen, die über ihren Serologiestatus informiert sind [23, 24]. Denn in relativ vielen Fällen erfahren Patientinnen/ Patienten erst beim Auftreten von Symptomen und durch den in diesem Zusammenhang durchgeführten Test, dass sie mit HIV infiziert sind [25]. Abgesehen davon, dass diese Patientinnen/Patienten nicht rechtzeitig die verfügbaren Therapien erhalten, könnten sie ihre Partner völlig unwissentlich weiterhin einem Infektionsrisiko aussetzen. Die Ärztinnen und Ärzte können zu einer Verbesserung der Situation beitragen, indem sie systematisch eine eingehende Risikoanamnese durchführen und gegebenenfalls einen freiwilligen Test vorschlagen.

Die hier vorgestellten Resultate sind mit gewissen Vorbehalten zu betrachten. Vor allem widerspiegeln sie wahrscheinlich eher das, was die Ärztinnen und Ärzten ihrer Ansicht nach im Rahmen ihrer Berufstätigkeit als notwendig erachten, als ihre tatsächlich tägliche Arbeit. Obwohl ein genauer Vergleich nicht möglich ist, muss in diesem Zusammenhang darauf hingewiesen werden, dass die Antworten der Ärztinnen und Ärzte nicht mit den Angaben der Schweizer Patientinnen/Patienten im Alter von 17 bis 45 Jahren zum Inhalt ihrer Konsultation(en) in den letzten zwölf Monaten vor der Studie übereinstimmen: Nur 6\% der Patientinnen/Patienten geben an, dass in diesem Rahmen ein Gespräch zum Thema HIV/Aids stattfand [26]. Dieser Anteil ist erheblich tiefer als die Anteile, die sich aus der vorliegenden Studie ergeben, in der mindestens 29\% der Ärztinnen und Ärzte angeben, routinemässig eine Anamnese des HIV-Risikos durchzuführen (Fall der getrennt lebenden Patientinnen/Patienten). Obwohl diese Verzerrung angesichts der Studienanlage unvermeidlich ist, lässt sich trotzdem beurteilen, wie sich die Ausweitung der Risikoabklärung auf die Gesamtbevölkerung entwickelt hat. Zudem lässt sich aufzeigen, dass weiterhin verschiedene Lücken und ein noch ungenutztes Schutzpotential bestehen.

Den Ärztinnen und Ärzten kommt im Bereich der Prävention eine vorrangige Rolle zu. Sie haben aufgezeigt, dass sie in der Lage sind, die erforderlichen Leistungen zu erbringen. Nun ist 
es wichtig, dass sie auf diesem Weg fortfahren, indem sie sich dafür einsetzen, eine noch grössere Zielgruppe zu erreichen, und indem sie durch die Wiederholung ihrer Befragungen und ihrer Beratung dauerhaft präventiv tätig sind.

\section{Literatur}

1 Bundesamt für Gesundheit, Eidgenössische Kommission für Aidsfragen. HIV-Prävention in der Schweiz: Ziele, Strategien, Massnahmen. Bern: Bundesamt für Gesundheit (BAG); 1993.

2 Somaini B, Twisselmann W, Ferber T, Schlegel F. HIV und Aids: Nationales Programm 1999 bis 2003. Bern: Bundesamt für Gesundheit (BAG); 1999.

3 Bundesamt für Gesundheit. Nationales HIV/AidsProgramm 2004-2008. Bern: Bundesamt für Gesundheit (BAG); 2003.

4 Grüninger U, Künzel M, Bosshard H. Verbindung der Schweizer Ärztinnen und Ärzte (FMH)/ Bundesamt für Gesundheit (BAG) (Hrsg.). HIVBeratung: Die ärztliche Beratung und Betreuung am Beispiel von HIV und Aids. Bern: Verbindung der Schweizer Ärztinnen und Ärzte (FMH); 1993.

5 Dubois-Arber F, Jeannin A, Meystre-Agustoni G, Moreau-Gruet F, Haour-Knipe M, Spencer B et al. Evaluation der Aids-Präventionsstrategie in der Schweiz im Auftrag des Bundesamtes für Gesundheit: fünfter zusammenfassender Bericht 1993-1995. Lausanne: Institut universitaire de médecine sociale et préventive; 1996. (Cah Rech Doc IUMSP, no. 120).

6 Meystre-Agustoni G, Dubois-Arber F, Gruninger U, Cassis I, Jeannin A. Pratiques et besoins des médecins suisses en matière de prévention de l'infection VIH au cabinet médical - synthèse des expériences, éléments de réflexion et recommandations. Schweiz Med Wochenschr 1995; 125(13):621-6.

7 Margolis AD, Wolitski RJ, Parsons JT, Gomez CA. Are healthcare providers talking to HIV-seropositive patients about safer sex? AIDS 2001; 15(17):2335-7.

8 Tao G, Branson BM, Anderson LA, Irwin KL. Do physicians provide counseling with HIV and STD testing at physician offices or hospital outpatient departments? AIDS 2003;17(8):1243-7.

9 Haidet P, Stone DA, Taylor WC, Makadon HJ. When risk is low: primary care physicians' counseling about HIV prevention. Patient Educ Couns 2002;46(1):21-9.

10 Ashton MR, Cook RL, Wiesenfeld HC, Krohn MA, Zamborsky T, Scholle SH, Switzer GE. Primary care physician attitudes regarding sexually transmitted diseases. Sex Transm Dis 2002;29(4):246-51.

11 Torkko KC, Gershman K, Crane LA, Hamman R, Baron A. Testing for Chlamydia and sexual history taking in adolescent females: results from a statewide survey of Colorado primary care providers. Pediatrics 2000;106(3):E32.

12 Ellen JM, Franzgrote M, Irwin CE, Jr., Millstein SG. Primary care physicians' screening of adolescent patients: a survey of California physicians. J Adolesc Health 1998;22(6):433-8.
13 Haley N, Maheux B, Rivard M, Gervais A. Lifestyle health risk assessment. Do recently trained family physicians do it better? Can Fam Physician 2000; 46:1609-16

14 Maheux B, Haley N, Rivard M, Gervais A. STD risk assessment and risk-reduction counseling by recently trained family physicians. Acad Med 1995;70(8):726-8.

15 Ewing GB, Selassie AW, Lopez CH, McCutcheon EP. Self-report of delivery of clinical preventive services by U.S. physicians. Comparing specialty, gender, age, setting of practice, and area of practice. Am J Prev Med 1999;17(1):62-72.

16 Lopez de Munain J, Torcal J, Lopez V, Garay J. Prevention in routine general practice: activity patterns and potential promoting factors. Prev Med 2001;32(1):13-22.

17 Haley N, Maheux B, Rivard M, Gervais A. Sexual health risk assessment and counseling in primary care: how involved are general practitioners and obstetrician-gynecologists? Am J Pub Health 1999; 89(6):899-902.

18 Kelts EA, Allan MJ, Klein JD. Where are we on teen sex?: Delivery of reproductive health services to adolescents by family physicians. Fam Med 2001;33(5):376-81.

19 Maheux B, Haley N, Rivard M, Gervais A. Do physicians assess lifestyle health risks during general medical examinations? A survey of general practitioners and obstetrician-gynecologists in Quebec. CMAJ 1999;160(13):1830-4.

20 Dubois-Arber F, Jeannin A, Spencer B, MeystreAgustoni G, Haour-Knipe M, Moreau-Gruet F et al. HIV/Aids-Prävention in der Schweiz: Bilanz und Ausblick. Schweiz Med Forum 2001;24:621-5.

21 Rosenbrock R, Dubois-Arber F, Moers M, Pinell P, Schaeffer D, Setbon M. The normalization of AIDS in Western European countries. Soc Sci Med 2000; 50(11):1607-29.

22 Dubois-Arber F, Jeannin A, Benninghoff F, Moreau-Gruet F, Spencer B. HIV/Aids: Zunehmendes Risikoverhalten? Das Prinzip der Vorsicht soll gelten. Bulletin des Bundesamtes für Gesundheit 2003;8:128-32.

23 Recommendations for incorporating human immunodeficiency virus (HIV) prevention into the medical care of persons living with HIV. Clin Infect Dis 2004;38(1):104-21.

24 Khonkarly MB, Merlani GM, Halfon P, Bellini C, Pelet A, Kazadi K, Cavassini ML, Tarr PE. Infection VIH: dix points clés pour la prise en charge par les praticiens. Med Hyg 2004;62:862-8.

25 Feuz M, Gebhardt, M. Time elapsing between the first positive HIV-test and AIDS diagnosis in Switzerland is decreasing [poster]. XIV International Conference on AIDS, Barcelona July 7-12 2002. Barcelona: International Aids Society (IAS); 2002. www.ias.se.

26 Dubois-Arber F, Jeannin A, Meystre-Agustoni G, Spencer B, Moreau-Gruet F, Balthasar H et al. Evaluation der HIV/Aids-Präventionsstrategie in der Schweiz: siebter Synthesebericht 1999-2003. Lausanne: Institut universitaire de médecine sociale et préventive; 2003. (Raisons de santé, 90b). www.hospvd.ch/iumsp. 